\title{
The Effects of Peer Interactions on the Development of Technological Fluency in an Early-Childhood, Robotic Learning Environment
}

\author{
Kevin J. Staszowski, Marina Bers \\ Tufts University/Tufts University
}

\section{Introduction}

Students interact in a classroom environment in a daily basis - interacting with teachers, classmates, and even with technology. The effects that these peer interactions within the educational setting have been extensively studied in the field of education over the past 20 years and have been shown to improve students self-esteem and attitude towards the educational process. ${ }^{7}$ Beyond these general improvements in students opinions on education and toward themselves, cooperative learning -the collaboration between peers to better understand unfamiliar topics - has been shown to positively influence a student development reading strategies ${ }^{6}$, computational skills in mathematics ${ }^{9}$, and a conceptual understanding of physics within education settings ${ }^{4}$. As such, the understanding of peer interactions has played an important role in the way curriculum is developed for the established fields of education.

With the relatively recent interest in expanding the educational curriculum frameworks to include concepts of engineering and technology, educators are looking for effective methods of addressing these new curriculum changes. However, engineering concepts are topics that are typically unfamiliar to teachers and few teacher have experience using educational technology related to engineering, like Lego Robolab program. ${ }^{2}$ The lack of acquaintance with engineering concepts and technology lead many educators to developing this curriculum for their classroom. One possible education aid that these educators might encourage, based on its record in advancing student progress in other disciplines, is cooperative learning within their students.

Little research exist that indicates what type of effect or even what type of interactions occur within a technological learning environment using engineering-based curriculum. The purpose of this study is to explore that question by determining the frequencies and effects of the peer interactions that occurred within an early-childhood robotic learning environment. Specifically, which of the underling concepts of the curriculum were most often used as the foundation of peer-interactions and how did those interactions appear to effect the children's understanding of engineering methods and processes.

Method

The study was conducted as part of a larger research study on the interactions between culture, technology, and family-member/child interactions. This larger study, Project Interactions, was a conceptual modification of a research project conducted by Bers and Urrea (2002) entitled Con-science. ${ }^{3}$ Con-science studied the effect of programmable Lego technology and parent-child interactions on the understanding of Jewish Culture in Argentina. Project 
Interaction changed the methodology developed in Con-science by using culture as a variable condition, rather than a constant.

The project consisted of a set of five workshops conducted between February and April of 2004. Two of the workshops were conducted on Saturdays, two were conducted on Sunday, and the remaining workshop was conducted on Tuesday and Thursdays mornings. The four workshops held on the weekends consisted of five two-hour session that generally occurred on non-consecutive weekends over the three month period. The morning workshops involved only children, while the afternoon workshops involved a family member/child teams. The weekday workshops consisted of twelve 45-minute sessions that occurred over six consecutive weeks.

Culture was integrated into the curriculum in the Sunday workshops and the workshop conducted during the week. In these workshops, participants took 30 to 45 minutes during the first three sessions to discuss and explore aspects of culture with the group. In the first session, the participants discussed what the definition was of or characteristics of culture - with the participants encouraged to share personal cultural traditions with the group. To facilitate this conversation, the participants were given a variety of children's books on the cultures to review prior to the discussion. In the second session, the participants discussed the relation between culture and food. This discussion culminated with the children creating cultural artifacts using breads from different cultures. In the third sessions on culture, the researchers asked the participants to reflect on the types of clothing worn in different culture. After this reflection, the participants crafted a piece of clothing using art supplies that expressed their personal culture.

All workshops involved programmable Lego bricks and the Robolab programming system as the primary technology. The first three sessions (or seven sessions for the weekday workshops) contained curriculum to help the participants become familiar with this technology, both building and programming. In the third session (or the eight session of the weekday workshop), the participants began working on the culminating project.

The workshops culminated with each participant or family-member/child team creating a technological artifact - or a robotic object that has some personal meaning to the participant or team of participants. Those participants who participated in the curriculum containing culture were required to make a technological artifact that connected with their cultural traditions. Participants worked on the artifact during the remaining workshop session. The weekend participants were allowed to take the artifacts home to work on and all participant had access to an instructor and extra building time during weeknight open-hours.

After building, programming, and decorating the artifact, the participants presented and digitally document their projects. The digital documentation took the form of mini-websites, where each participant was asked to compose and type a small description of their project. This description would accompany photographs of their final project. The students were asked to include in their composition a description of what they build for their final project, how they built it, what was something in the workshop that was challenging to them, and what they learned in the workshop. These mini-websites were combined by workshop and uploaded onto Project Interactions website (http://ase.tufts.edu/dev_tech/Project_Inter-Actions) for general review. 
The workshops were taught by instruction teams made up of undergraduate and graduate students from the Tufts Department of Child Development or the Tufts School of Engineering. Each instruction team consisted of the same head teacher and three assistant teachers throughout the workshop. One instruction team was responsible for both of the Saturday workshops, another for both of the Sunday workshops, and third instruction team handled the weekday workshops.

Each instruction team used the same set of curriculum for each workshop. The curriculum was designed to introduce the workshop participants to general engineering methods and processes. These methods and processes were formulated by the research team and were based on the team's previous experience with engineering curriculum, the Massachusetts Curriculum Frameworks for Science and Engineering/Technology, and the limitations of the Lego/Robolab technology. The research team defined the methods of engineering as the ability to integrate curriculum concepts into personal constructions or the knowledge of what to do with the materials. The methods were reflected the participants' interactions with both the building component and the programming component of the Lego/Robolab technology. The processes of engineering were defined as the ability to develop a strategy to follow to complete a design task and the ability to express an understanding the purpose behind the application of a curriculum concept. The researchers envisioned the process of engineering as the knowledge of why a certain method or concept was used.

The curriculum was modified for each workshop to address needs of the participants in each workshop -as those needs were identified by the instruction team. Regardless of the instruction team, all the workshops were taught using a constructionist/constructivist theoretical framework. This framework rests on the idea that individuals learn better when the individuals take an active role in the construction of knowledge. ${ }^{5}$ Rather than lecture the participants or give the participants specific design tasks to mimic, the instruction team presented the workshop participants with broad design challenges. Members of the instruction team then worked one-onone with participants as the participants developed solutions to the design challenges; the participants were also encouraged to work with other workshop participants. Each workshop session culminated with a technology circle, where each participant would be able to present his or her creation to the group.

Method of Analysis

The study population consisted of the 17 children participating in the weekday workshops. This workshop was part of a 1 st and 2 nd grade, mixed-aged class at the laboratory school located within the Eliot-Pearson Department of Child Development. The workshop was integrated in the normal schedule of the class to replace the science period and, for this reason, this group of children was chosen for the study population. The purpose of this study was the exploration of how peer interactions might affect curriculum used in a classroom setting and the weekend workshops were extracurricular activities that the participants chose to attend.

Culture was also not consider during the analysis of the peer interactions, despite the integration of culture into the curriculum used during the weekday workshops. The classroom was culturally homogeneous, which resulted in few animated discussion regarding culture. 
Culture was also only moderately expressed by the children in their final project for the workshop.

To identify the peer interactions occurring within each of these environments, the researcher reviewed video footage taken during the workshops and field notes taken by the instruction team. The researchers used this data to identify peer interactions between the children that related to the methods and processes of engineering that underlined the Project Interaction curriculum. The peer interactions identified in the workshops were broken into the following categories

- Design - A design interaction occurred when the students were speaking about a method to be used to solve a design problem. This interaction was considered part of the process of engineering.

- Building - A building interaction occurred when a child interacted with another child while building. This interaction was localized to the specific structure that the child was working in. This interaction was considered part of the method of engineering.

- Building Concepts - A building concept interaction occurred when a child explained or tried to explain a concept of building to another child. This interaction is different than the building interaction because the child is making a more generalize statement that expresses a curriculum concept. This interaction was considered part of the process of engineering.

- Programming - A programming interaction occurred when children help each other with some mechanic of the physical aspect of Robolab programming. This interaction involves identifying errors in the programming structure, or pointing out an incorrect method of downloading the program. This interaction was considered part of the method of engineering.

- Programming Concepts - A programming concept was defined as more a curriculum concept related to the Robolab programming language. An example of this interaction would be a children discussing how a loop function worked in the Robolab programming environment. This interaction was considered part of the process of engineering.

Within each of these five categories, the researchers also identified the direction of the communication between the children as either two-way or one-way. A child interacting using two-way communication would both ask and respond to questions with peer. The children would use the discussion as a means for them both to better understand the issue the sparked the communication. A child interacting with one-way communication would relate to a peer the information necessary to solve a problem, but would not elaborate on the information or ask questions of the peer about the problem. The child is giving or receiving answers with providing or asking for any explanation for that answer. Within cooperative learning environments, research has shown that engaging in two-way communication while interacting with peers leads to better understand of a topic by both the parties involved in the communication. ${ }^{8}$ 
To identify the children's understanding of the methods and processes of engineering used in the curriculum, a review was conducted of the projects written by the children as part of the digital documentation process. The researchers believed that the salient or important aspects of the workshop would be expressed in the documentation process. From the analysis of these descriptions, the researchers tabulated the instances of children writing about designing their project, building their project, concept of building that they learned through building their project, programming their projects, and concepts of programming they learned through the building of their project.

Results

Table 1 indicates the frequencies of specific peer interaction that occurred within the workshop related to the general categories above.

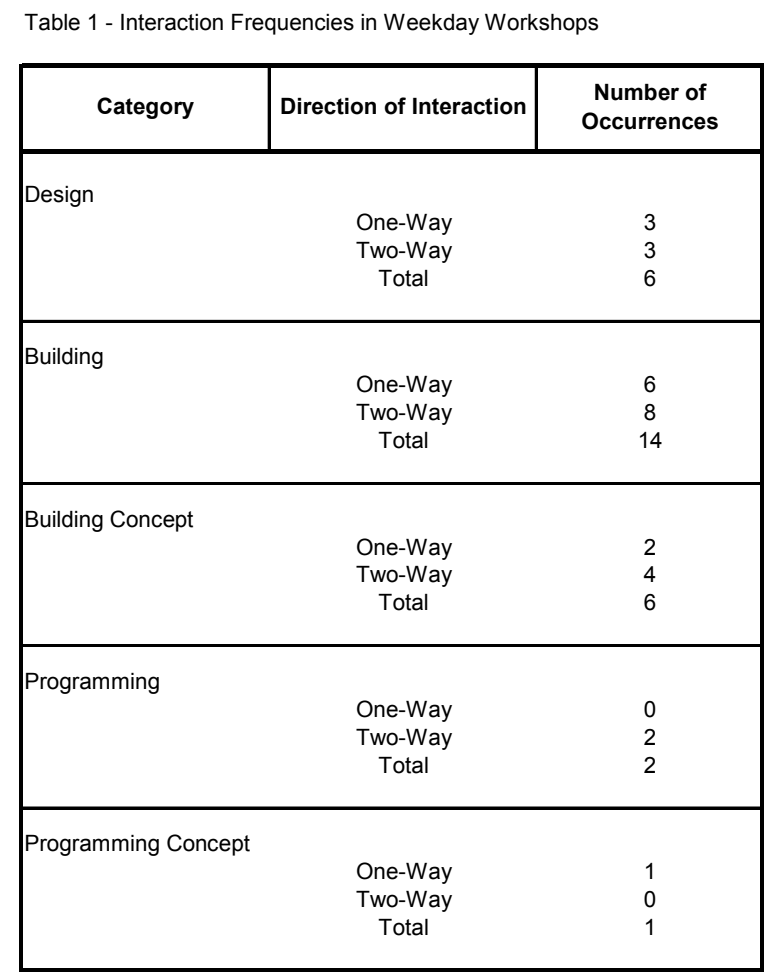

The most common type of peer interaction that occurred within the workshop was regarding building with the technology, while the least frequent form of peer interactions were those related to programming concepts. The children in the workshop also tended to engage in two-way interactions much more often than in one-way interactions.

Table 2 shows the number of instances that the children wrote about topics indicative to the five categories of curriculum concepts used to describe the peer interactions in the workshop. 
Table 2 - Frequence of Language in Students' Final

Project Documentation related to Categories of Peer Interactions

\begin{tabular}{|l|c|}
\hline \multicolumn{1}{|c|}{ Category } & Number of Occurrences \\
\hline Design & 6 \\
Building & 11 \\
Building Concepts & 2 \\
Programming & 8 \\
Programming Concept & 1 \\
\hline
\end{tabular}

From the review of the students' digital documentation of their final projects, the children wrote more about building with the Lego technology than about any other portion of the workshop. The children also wrote about significantly about programming with the technology, but much of this dialogue discussed how an instructor programmed the students' project - rather than the student taking personal responsibility for the programming.

\section{Discussion}

The purpose of this study was to determine the frequencies and effects of the peer interactions that occurred within an early-childhood robotic learning environment. The general findings of this study indicated that most frequent peer interaction occurring within this learning environment revolves around building with the technology. The effects of these peer interactions also appear to influence the important topics remembered by the children when the children reflect on curriculum completed during the workshop.

The strong concentration of peer interaction related to building reflects the topic most interesting and most challenging to the students. Peer interactions often occur when children are attempting to gain a better understanding of an educational concept. In this workshop experience, many students had difficulty building with the technology - despite the children's expressed familiarity with the technology. The peer interactions are another indicator that the limitations of dealing with the basics of the technology may have prevented the children from interacting about the engineering processes that underlies the curriculum. However, the children did express that building was the most memorable part of the workshop process, so the children appeared to be actively engaging in engineering methodology. An outside facilitator may be required to help move the conversations conducted between peers into a realm related closer to engineering processes.

The frequency of two-way interactions within this learning environment may be indicative to the classroom expectation that the children. Two-way interactions indicate that the children were engaging in the process of learning about the technology that benefited both parties involved. The continued encouragement of peer interaction in technological learning environments is beneficial for those educators who feel uncomfortable with the technology or engineering concepts being taught. The two-way interaction should allow the students within the learning environment to begin reflect on confusions in their understanding of topics and also formulate a mindset to clarify those confusions - without the intervention of the teacher.

The study implication should be considered in terms of the limitations of the study. The major internal limitation to the study is that the workshops curriculum generally focused the majority of instruction time on building and building concept as the curriculum evolved to reflect 
the children's interests. Without the same degree of exposure to programming, the children may not have had the understanding of this technology to engage in peer interactions around the subject. The interactions considered within this study are those that are directly relate to the curriculum foundation of Project Interactions that were recorded on the digital footage taken in the workshops. Other forms of peer interaction within the classroom may have been occurring outside that fell outside the categories established by the researchers that may be indicative of additional peer interactions or of learning that does not directly relate to engineering or technology curriculum.

Overall, the implications for this study include a better understanding of the frequencies of curriculum-related peer interactions within a robotic learning environment and the benefits of creating group-based learning projects for the use of technology.

References

1 Bers, M. New, B. Boudreau, L (2004) Teaching and Learning when No One is Expert: Children and Parents explore Technology. Early Childhood Research \& Practice. 6(2)

2 Bers, M. Portsmore, M. (In Press) Teaching Partnerships: Early childhood and engineering students teaching math and science through robotics. Journal of Science Education and Technology

3 Bers, M. Urrea, C (2000).Technological Prayers: Parents and Children Working with

Robotics and Values. In Robots for Kids: Exploring New Technologies for Learning

Experiences. Edited by A. Druin \& J. Hendler. NY: Morgan Kaufman. pp. 194-217

4 Howe, C., Tolmie, A., Greer, K., \& McKenzie, M. (1995). Peer collaboration and conceptual growth in physics: Task influences on children's understanding of heating and cooling. Cognition and Instruction. 13. 483-503.

5 Papert, S (1993) Mindstorms Children, Computers, and Powerful Ideas. Perseus Publishing. Cambridge, Massachusetts.

6 Rojas-Drummond, S. Hernadez, G. Velez, and M. Villagran, G (1997) Cooperative Learning and the Appropriation of Procedural Knowledge by Primary School Students. Learning and Instruction. 8(1) 37-61

7 Stevens, R. Slavin, R (1995) The Cooperative Elementary School: Effect's on Student Achievement, Attitudes, and Social Relations. American Educational Research Journal. 15. 321-351

8 Webb, N. Farivar, S (1994) Promoting Helping Behavior in Cooperative Small Groups in Middle School Mathematics. American Journal of Educational Research. 31(2) 369-395

9 Webb, N. Troper, J. Fall, R. (1995) Constructive Activity and Learning in Collaborative Small Groups. Journal of Educational Psychology. 87(3) 406-423 
KEVIN STASZOWSKI is a masters candidate at the Eliot-Pearson Department of Child Development at Tufts University. His research focuses on the effect that technology and teaching style has on young children's performance on standardized tests and on cognitive and metacognitive processes.

MARINA BERS is an assistant professor at the Eliot-Pearson Department of Child Development at Tufts University. She completed her Ph.D. in 2001 at the MIT Media Laboratory. Her research involves the design and study of "identity construction environments," technological learning tools to support children's exploration of identity through the creation of a participatory community. 